\title{
SURGICALLY TRANSFORMING LINKS INTO NONINVERTIBLE KNOTS
}

\author{
BY WILBUR WHITTEN \\ Communicated by M. L. Curtis, July 14, 1971
}

The difficulties manifested by invertibility problems of knots have long plagued knot theorists. It was, in fact, not known until 1963 that any noninvertible knots existed. In that year, H. F. Trotter exhibited his now famous noninvertible pretzel knots [2]. There are still no reasonable "invertibility tests" which might be applied to particular examples. Indeed, Trotter's pretzel knots together with some others which he has since found (unpublished) have remained the only known examples of noninvertible knots. Our basic aim in this paper is to announce a surgical process for generating noninvertible knots in profusion and variety.

In his paper, Trotter asked the following interesting question: Do there exist prime, amphichaeral, noninvertible knots? We announce here an affirmative answer to this question and present two examples : one (Figure 3 ) is +amphichaeral, while the other (Figure 4) is - amphichaeral.

1. Noninvertible knots from links. An oriented, ordered link $L=K_{1} \cup K_{2}$ of two components tamely imbedded in the oriented 3-sphere $\mathfrak{S}$ will be called admissible, provided it satisfies the following two conditions:

(i) The component $K_{1}$ is of trivial knot type;

(ii) the order [1] with respect to $K_{2}$ of $W$ is greater than zero, where $W$ is the closure of the complement in $\subseteq$ of a tubular neighborhood $\mathfrak{B}$ of $K_{1}$ in $\mathfrak{\Im}-K_{2}$; that is, $L$ is unsplittable.

Consider an admissible link $L=K_{1} \cup K_{2}$, denote by $W$ the closure of the complement in $\Im$ of a tubular neighborhood of $K_{1}$ in $\Im-K_{2}$, and let $V$ denote a knotted, closed solid torus tamely imbedded in $\subseteq$. Let $K$ be a core of $V$. The (oriented) image $\mathscr{K}$ of $K_{2}$ under a faithful homeomorphism (takes meridians to meridians and longitudes to longitudes) of $W$ onto $V$ will be called a surgical transmute (or simply a transmute) of $L$ with respect to $V$, and we shall say that $\mathscr{K}$ was obtained from $L$ by surgical transmutation.

We may generate a megaloriety of noninvertible knots by surgical transmutation and applications of the following theorem.

AMS 1970 subject classifications. Primary 55A25, 55A05, 55A10; Secondary 57A10.

Key words and phrases. Noninvertible knots and links, surgical transmutation, companion knots, prime knots, plus and minus amphichaeral.

Copyright (C) American Mathematical Society 1972 


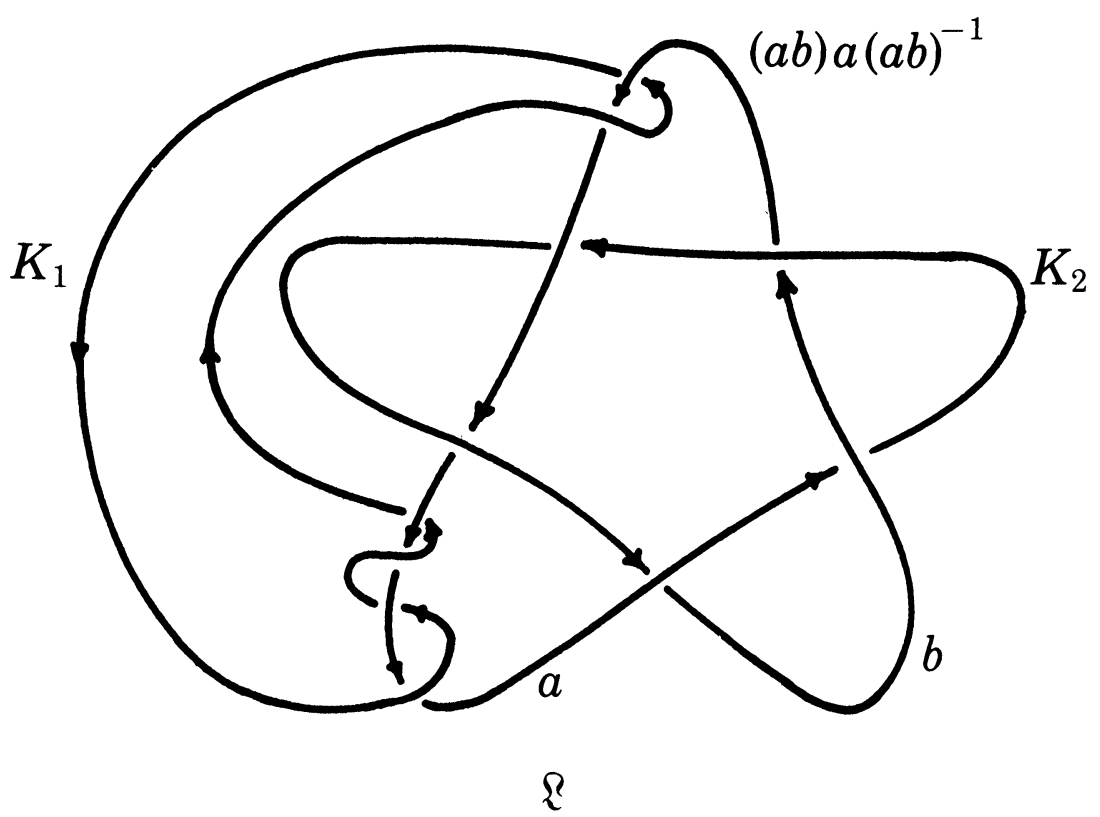

FIGURE 1

THEOREM 1. Let $L=K_{1} \cup K_{2}$ be an admissible link in $\subseteq$ and suppose that $\mathscr{K}$ is a surgical transmute of $L$ with respect to the solid torus $V$ with core $K$. Let $\alpha$ denote the order of $V$ with respect to $\mathscr{K}$, and let $w$ denote the winding number [1] of $\mathscr{K}$ in V. Furthermore, suppose:

(a) $K$ (not oriented) is a companion of $\mathscr{K}$ (not oriented), and there is no system of two or more (tame) solid tori $V_{1}, \ldots, V_{n}$ in $\mathfrak{S}_{\text {each }}$ containing $\mathscr{K}$ in its interior with winding number $w$ and order $\alpha$, such that the closure of the complement in $\mathfrak{S}$ of each $V_{i}$ belongs to the interior of each of the others, and the core of each $V_{i}$ is of the same knot type as $K$;

(b) $L$ does not admit the symmetry $(1,1,-1,(1))[4]$.

It follows that if $L$ is also noninvertible, then so is $\mathscr{K}$. Moreover, if $L$ does not admit a symmetry of the form $\left(-1, \varepsilon_{1}, \varepsilon_{2},(1)\right)$, then $\mathscr{K}$ is not amphichaeral.

The proof of Theorem 1 (based extensively on results of $\mathrm{H}$. Schubert in [1]) will appear in another journal, together with proofs of the theorems announced in $\$ 2$.

2. Noninvertible knots: examples.

THEOREM 2. The link $\mathfrak{L}$ of Figure 1 is admissible and the class $\mathfrak{C}$ of surgical transmutes of $\mathfrak{L}$ contains a representative from each of an infinite collection 


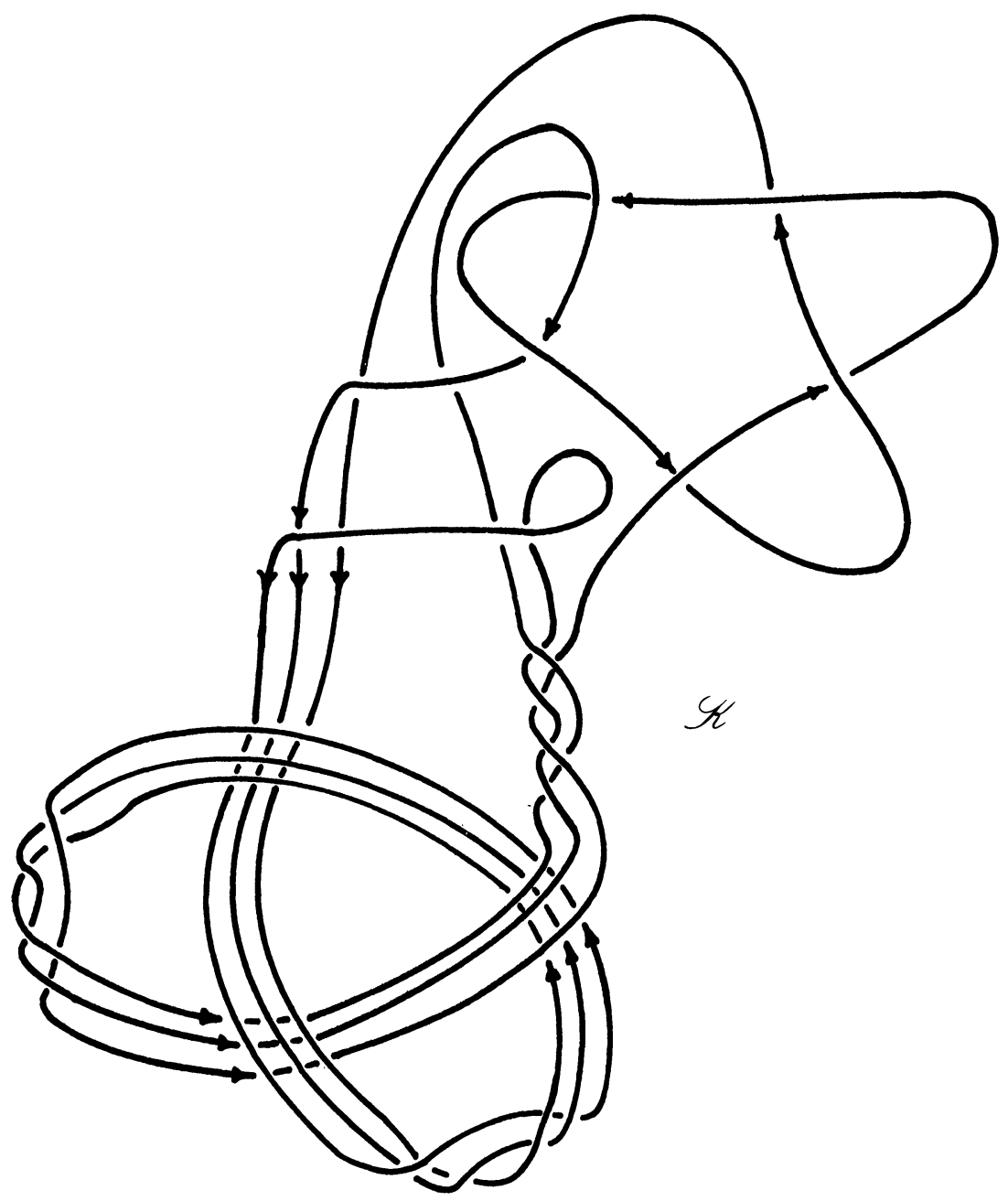

FigURE 2

of distinct knot types. Moreover, each knot in $\mathbb{C}$ is both noninvertible and nonamphichaeral.

The knot $\mathscr{K}$ of Figure 2 is a simple example of a transmute of $\mathfrak{L}$.

THEOREM 3. The knot $\mathscr{K}_{(+)}\left(\mathscr{K}_{(-)}\right)$of Figure $3(4)$ is prime, noninvertible, and $+(-)$ amphichaeral. 


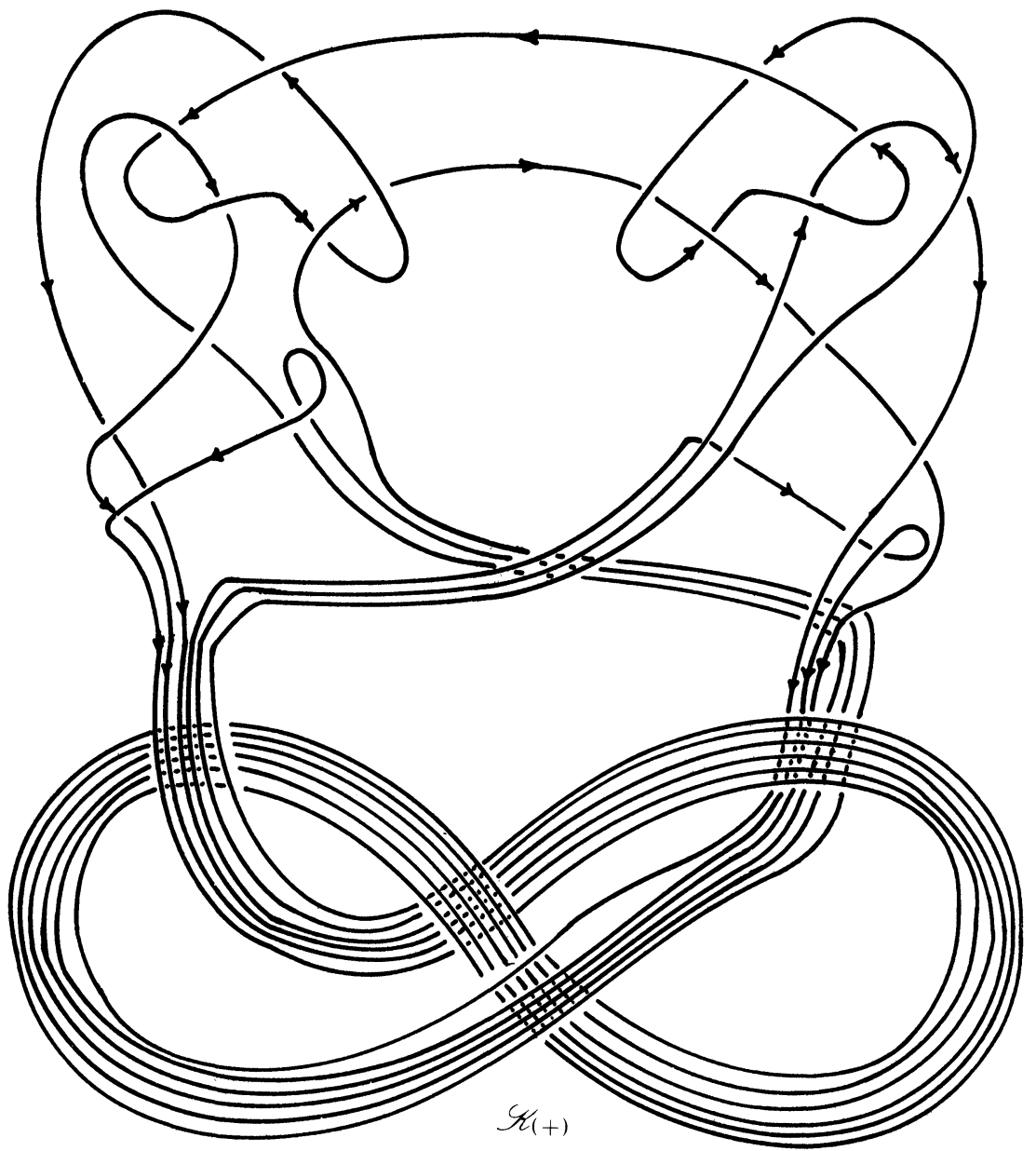

FIGURE 3

It is not difficult to determine the links $\mathfrak{L}_{(+)}$and $\mathfrak{R}_{(-)}$of which $\mathscr{K}_{(+)}$and $\mathscr{K}_{(-)}$, respectively, are surgical transmutes. The methods and results of [3] and [1] can be used to construct proofs of Theorems 2 and 3.

\section{REFERENCES}

1. H. Schubert, Knoten und Vollringe, Acta. Math. 90 (1953), 131-286. MR 17, 291. 


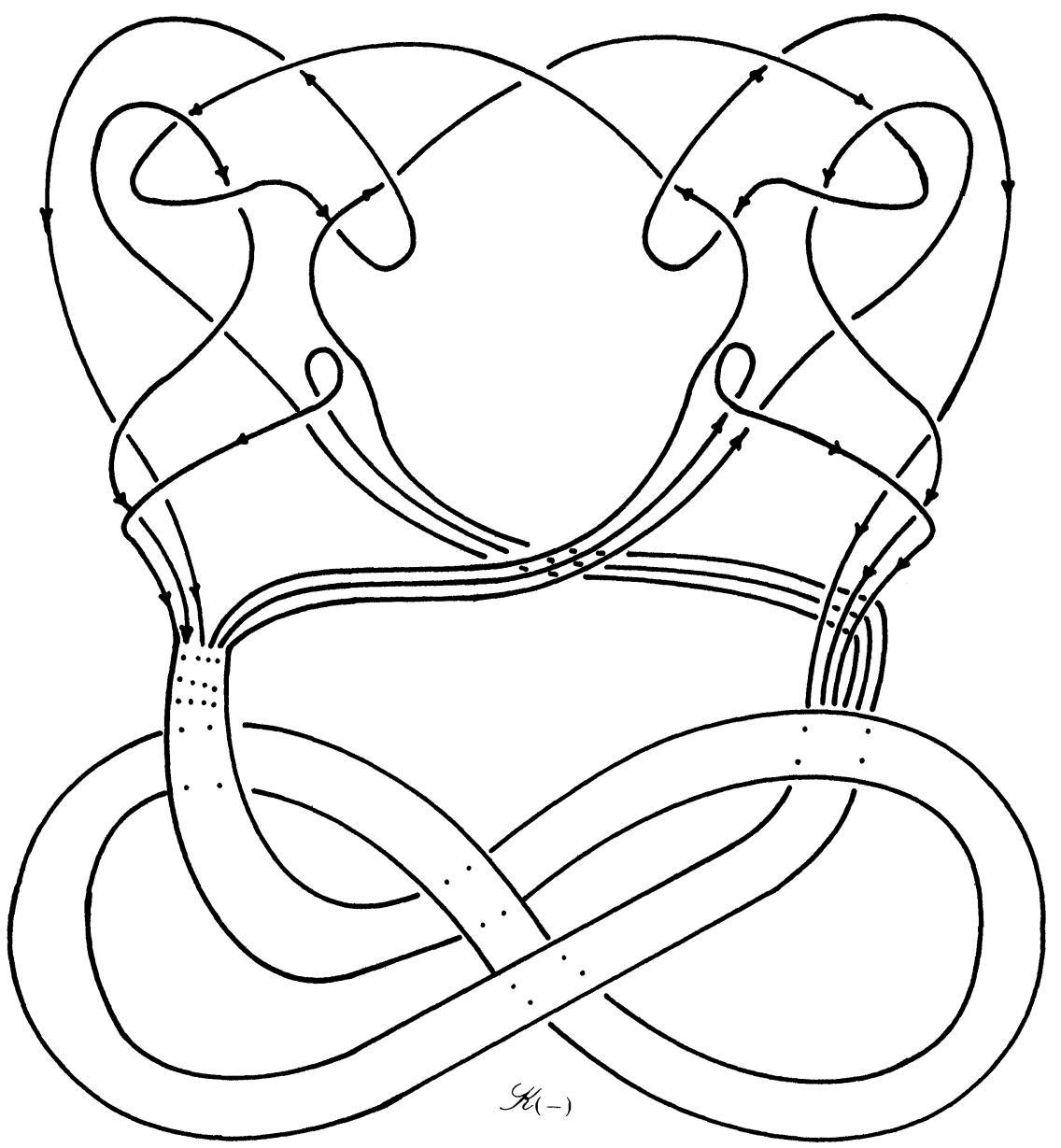

FIGURE 4

2. H. F. Trotter, Non-invertible knots exist, Topology 2 (1963), 275-280. MR 28 \# 1618; errata, 30, 1205.

3. W. C. Whitten, Jr., A pair of non-invertible links, Duke Math. J. 36 (1969), 695-698. MR 40 \#6532.

4. — On the link-symmetric group, Topology of Manifolds (Proc. the Univ. of Georgia Inst., 1969), Markham, Chicago, Ill., 1970, pp. 212-213.

Department of Mathematics, University of Southwestern Louisiana, Lafayette, LOUISIANA 70501 